\title{
A pilot randomised clinical trial of mepolizumab in COPD with eosinophilic bronchitis
}

\author{
To the Editor:
}

Airflow limitation in chronic obstructive pulmonary disease (COPD) is associated with influx of various inflammatory cells (e.g. eosinophils, neutrophils, lymphocytes, macrophages) into the airways. Approximately one-third of stable COPD patients and one in five COPD exacerbations are associated with eosinophilic bronchitis that usually responds to inhaled or ingested corticosteroids [1]. Specific anti-eosinophil agents like mepolizumab, a humanised monoclonal antibody against interleukin 5 (IL-5), reduce severe asthma exacerbations and improve lung function [2-4]. The improvement in forced expiratory volume in $1 \mathrm{~s}(\mathrm{FEV} 1)$ is also associated with a decrease in biomarkers of airway remodelling, such as sputum hyaluronan and versican, over a 6-month treatment period [5]. It is not known if the same benefits are observed in patients with COPD and eosinophilia in whom the airflow obstruction is due to cigarette smoke-related bronchitis and emphysema.

The primary objective of this "proof of principle", single centre, randomised, placebo-controlled, parallelgroup, double-blinded, 6-month trial (with 4-month follow up) was to determine if mepolizumab could decrease sputum eosinophil percentage in those patients with cigarette smoke-related COPD who have persistent sputum eosinophilia. The other objectives were to assess the effects of mepolizumab on blood eosinophil count, lung function, exacerbation rate, symptoms and quality of life, and sputum hyaluronan and versican as markers of airway remodelling. The study enrolled adults aged $40-80$ years, with moderate to severe COPD (post-bronchodilator FEV 1 to forced vital capacity ratio $<70 \%$ and $\mathrm{FEV} 1<60 \%$ predicted, on high doses of inhaled corticosteroids and long-acting bronchodilator $\beta$-agonists, muscarinic agonist or both) and at least one major exacerbation requiring prednisone in the preceding year and who had demonstrated, within the past 24 months, at least $100 \mathrm{~mL}$ improvement in FEV1 with a 5-day course of $30 \mathrm{mg}$ prednisone daily. Participants were current or past smokers with a tobacco smoking history of $\geqslant 10$ pack-years with sputum eosinophils $>3 \%$, or many (3+) free eosinophil granules at randomisation and on at least one occasion in the past 2 years (table 1). Patients with a previous diagnosis of asthma were not excluded if they also had current COPD. 16 patients had qualitative computed tomography evidence of emphysema (10 centrilobular, 2 paraseptal, 4 combined centrilobular and paraseptal); quantitatively, all patients had evidence of some emphysema (mean \pm SD of emphysema score $6 \pm 8 \%$ ). Sputum was induced and processed according to the method described by Pizzichini et al. [6] and hyaluron and versican was measured by ELISA [5]. Pulmonary function was assessed as recommended by the American Thoracic Society/European Respiratory Society guidelines. Symptoms and quality of life was assessed using the Chronic Respiratory Questionnaire and the COPD Assessment Test. Inspiratory and expiratory high-resolution computed tomography images of the thorax were obtained on a GE 64-slice scanner and analysed using Pulmonary Workstation 2.0 (VIDA Diagnostics, Inc., Coralville, IA, USA). Patients received monthly infusions of mepolizumab $(750 \mathrm{mg})$ or placebo in addition to their inhaled medications. They were monitored every month and at the time of an exacerbation. The study was approved by Hamilton Integrated Institutional Review Board and by Health Canada. All patients provided written informed consent. Between and within group intention-to-treat analyses were performed using SPSS (version 19, SPSS Inc., Chicago, IL, USA).

18 patients (13 males, mean age 66 years, mean smoking history 39 pack years, mean body mass index $\left.28 \mathrm{~kg} \cdot \mathrm{m}^{-2}\right)$ were randomly assigned to receive mepolizumab $(\mathrm{n}=8)$ or placebo $(\mathrm{n}=10)$. One patient (from placebo group) left the study just after randomisation because of severe exacerbation requiring hospitalisation.

@ERSpublications

Eosinophils may not directly contribute to luminal obstruction in COPD although they may predict steroid response http://ow.ly/b3Au308wA0m

Cite this article as: Dasgupta A, Kjarsgaard M, Capaldi D, et al. A pilot randomised clinical trial of mepolizumab in COPD with eosinophilic bronchitis. Eur Respir J 2017; 49: 1602486 [https://doi.org/ 10.1183/13993003.02486-2016]. 
TABLE 1 Comparison between groups (mepolizumab versus placebo) in the intention-to-treat analysis

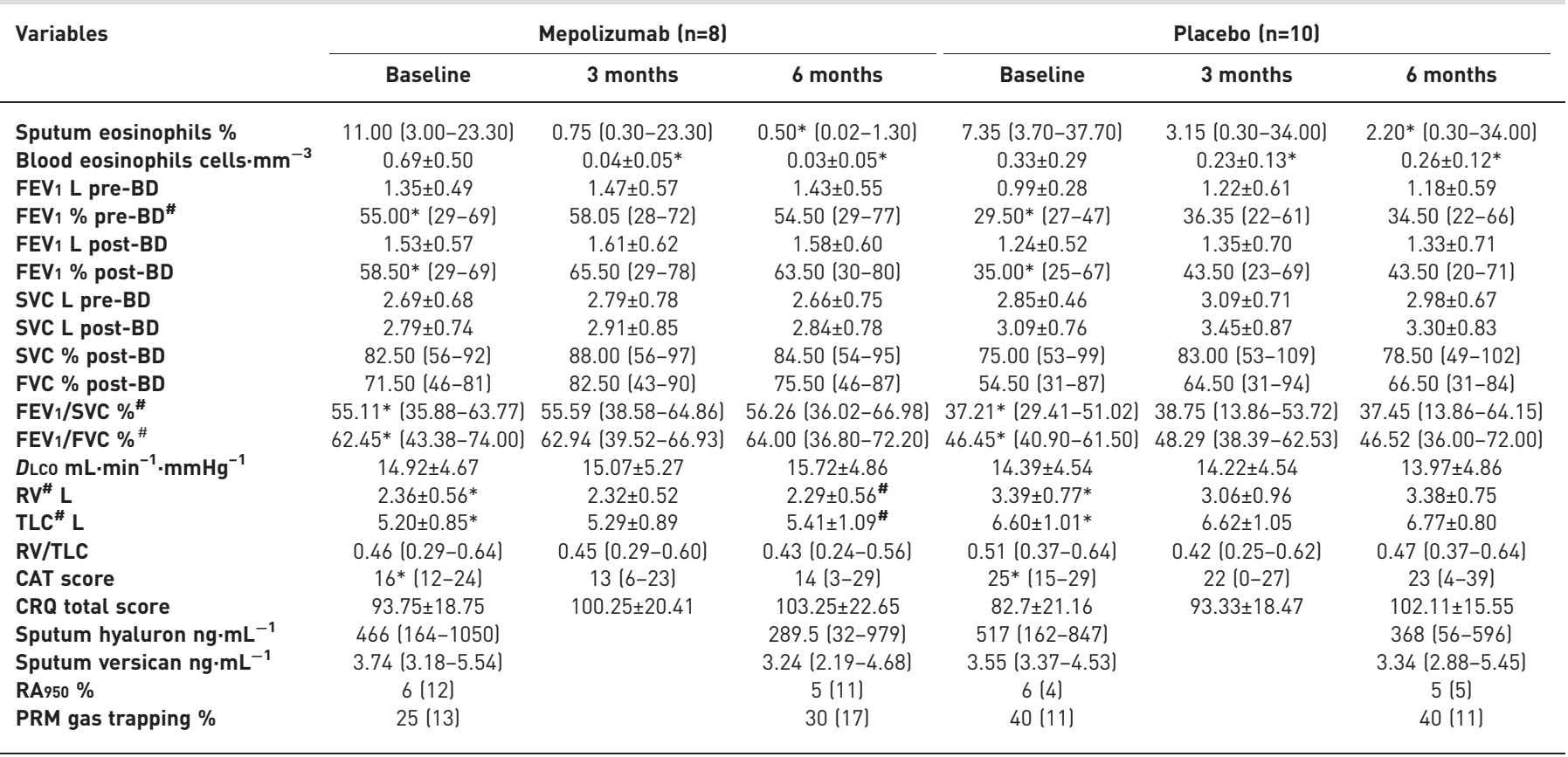

Data are presented as mean \pm SD or medium (range). FEV1: forced expiratory volume in $1 \mathrm{~s}$; BD: bronchodilator; SVC: slow vital capacity; FVC: forced vital capacity; DLCO: diffusing capacity of the lung for carbon monoxide; RV: residual volume; TLC: total lung capacity; CAT: COPD Assessment Test; CRQ: Chronic Respiratory Questionnaire; RA950: relative area on high-resolution computed tomograpgy image < 950 Hounsfield units; PRM: parametric response maps. There are significant differences between the two groups in the 6-month sputum eosinophil $\%$ and in blood eosinophil counts at 3 and 6 months. There are baseline differences between groups in pre-BD FEV $1 \%$, post-BD FEV $1 \%$, $\mathrm{FEV}_{1} / \mathrm{FVC}, \mathrm{FEV} 1 / \mathrm{SVC}, \mathrm{RV}, \mathrm{TLC}$ and CAT values. These were accounted for in the analysis by using ANCOVA. *: significant difference between two groups (mepolizumab versus placebo); " : analysis by ANCOVA to adjust for baseline differences.

Mepolizumab reduced sputum eosinophil counts from $11.0 \%$ at baseline to $0.5 \%$ at 6 months and blood eosinophils from $0.7 \pm 0.5$ cells $\cdot \mathrm{mm}^{-3}$ at baseline to $0.03 \pm 0.05$ cells $\cdot \mathrm{mm}^{-3}$ at 6 months. Corresponding numbers in the placebo group were $7.4 \%$ at baseline to $2.2 \%$ for sputum eosinophils $(\mathrm{p}<0.05)$ and 0.33 \pm 0.29 cells $\cdot \mathrm{mm}^{-3}$ to $0.26 \pm 0.12$ cells $\cdot \mathrm{mm}^{-3}$ in the placebo group $(\mathrm{p}<0.05)$. The change in sputum eosinophil percentage and blood eosinophil count from baseline to 6 months was significantly different between groups. However, there were no significant differences between the mepolizumab and placebo groups in the pre- and post-bronchodilator spirometry measures, residual volume (RV), total lung capacity (TLC), RV/TLC, diffusing capacity of the lung for carbon monoxide values, sputum hyaluron, versican or radiological evidence of remodelling $(n=6)$, or patient related outcomes at 3 and 6 months (table 1). The mean changes from baseline in these parameters were also not significantly different between the two groups. Four out of eight patients in the mepolizumab group and seven out of 10 patients in the placebo group experienced an exacerbation during the 6-month study period (50\% versus $70 \%, \mathrm{p}>0.05)$ while four patients in the mepolizumab arm and one patient in the placebo arm exacerbated during the follow-up period.

The findings of our study are very similar to the only other anti-IL-5 study in COPD where benralizumab, a monoclonal antibody to IL-5 receptor $\alpha$, depleted blood and sputum eosinophils, produced a trend towards improvements in pre-bronchodilator and post-bronchodilator FEV1 and St George's Respiratory Questionnaire for COPD patients score but did not reduce exacerbations [7]. This indicates that unlike in asthma, the role of eosinophils in COPD is complex. Sputum [8-10] and blood eosinophilia in COPD [11] are predictors of improvement in $\mathrm{FEV}_{1}$ and reduction in exacerbation rates following treatment with inhaled or ingested corticosteroids [12]. Despite a mean historic improvement in FEV1 of $356 \mathrm{~mL}$ with a 5 -day course of $30 \mathrm{mg}$ daily prednisone, we were not able to observe the same magnitude of improvement in FEV1 with 6 monthly infusions of $750 \mathrm{mg}$ mepolizumab. This suggests that the improvement with corticosteroids in COPD (often used along with long-acting $\beta$-agonists or anticholinergics) may be based on its actions on pathways or cells other than the eosinophils, but those that may be increased along with the eosinophils, such as the helper or suppressor T-lymphocytes or B-lymphocytes [13], macrophages [14] or metachromatic cells such as the basophil or mast cell [15]. This requires further evaluation.

The major limitation of this study is that some participants in the placebo group showed unexpected changes in sputum eosinophil counts, pre-bronchodilator FEV1, pre- and post-bronchodilator vital capacity 
and in sputum hyaluronan and versican levels. These participants denied any change of medications or steroid use during the study period. Further, careful scrutiny of their pharmacy dispensing logs did not identify any unauthorised use of corticosteroids. Therefore, the only likely explanation for this is improved compliance to medications during the clinical trial as a result of frequent and careful follow-up. We faced recruitment challenges forcing us to restrict our sample size, which reduced the study power to approximately $60 \%$ (effect size $4.21 \%$ with an alpha of 0.1 ).

In summary, we report two novel observations: first, a history of cigarette smoking or associated emphysema does not reduce the anti-eosinophil effect of mepolizumab in COPD. Secondly, the decrease in sputum and blood eosinophil count were not associated with significant improvements in other outcomes such as lung function parameters, exacerbation rates, sputum markers of remodelling and health-related quality of life scores. This suggests that eosinophils when present in the airway, unlike in asthma, may not play a major pathobiological role in patients with smoking-related COPD because of smoker's bronchitis or emphysema.

Angira Dasgupta $^{1}$, Melanie Kjarsgaard ${ }^{1}$, Dante Capaldi ${ }^{2}$, Katherine Radford ${ }^{1}$, Fernando Aleman ${ }^{1}$, Colm Boylan ${ }^{3}$, Leonard C. Altman ${ }^{4}$, Thomas N. Wight ${ }^{5}$, Grace Parraga ${ }^{2}$, Paul M. O'Byrne ${ }^{1}$ and Parameswaran Nair ${ }^{1}$

${ }^{1}$ Dept of Medicine, McMaster University and St Joseph's Healthcare, Hamilton, ON, Canada. ${ }^{2}$ Robarts Research Institute, Western University, London, ON, Canada. ${ }^{3}$ Radiology, McMaster University \& St Joseph's Healthcare, Hamilton, ON, Canada. ${ }^{4}$ University of Washington, Seattle, WA, USA. ${ }^{5}$ The Matrix Biology Program, Benaroya Research Institute at Virginia Mason, Seattle, WA, USA.

Correspondence: Parameswaran Nair, Firestone Institute for Respiratory Health, St Joseph's Healthcare, 50 Charlton Avenue East, Hamilton, Ontario, L8N 4A6, Canada. E-mail: parames@mcmaster.ca

Received: Nov 142016 | Accepted after revision: Dec 202016

Clinical trial registration: NCT01463644.

Support statement: This study was funded by the Canada Research Chair program and GlaxoSmithKline. Funding information for this article has been deposited with the Open Funder Registry.

Conflict of interest: Disclosures can be found alongside this article at erj.ersjournals.com

\section{References}

1 D'silva L, Hassan N, Hong W, et al. Heterogeneity of bronchitis in obstructive airway diseases in a tertiary clinic. Can Respir J 2011; 18: 144-148.

2 Nair P, Pizzichini MM, Kjarsgaard M, et al. Mepolizumab for prednisone-dependent asthma with sputum eosinophilia. N Engl J Med 2009; 360: 985-993.

3 Haldar P, Brightling CE, Hargadon B, et al. Mepolizumab and exacerbations of refractory eosinophilic asthma. N Engl J Med 2009; 360: 973-984.

4 Flood-Page P, Menzies-Gow A, Phipps S, et al. Anti-IL-5 treatment reduces deposition of ECM proteins in the bronchial sub-epithelial basement membrane of mild atopic asthmatics. J Clin Invest 2003; 112: 1029-1036.

5 Ayars AG, Altman LC, Potter-Perigo S, et al. Sputum hyaluronan and versican in severe eosinophilic asthma. Int Arch Allergy Immunol 2013; 161: 65-73.

6 Pizzichini E, Pizzichini MM, Efthimiadis A, et al. Measurement of inflammatory indices in induced sputum: effects of selection of sputum to minimize salivary contamination. Eur Respir J 1996; 9: 1174-1180.

7 Brightling CE, Bleecker ER, Panettieri RA Jr, et al. Benralizumab for chronic obstructive pulmonary disease and sputum eosinophilia: a randomised, double-blind, placebo-controlled, phase 2a study. Lancet Respir Med 2014; 2: 891-901.

8 Brightling CE, Monteiro W, Ward R, et al. Sputum eosinophilia and short-term response to prednisolone in chronic obstructive pulmonary disease: a randomized controlled trial. Lancet 2000; 356: 1480-1485.

9 Pizzichini E, Pizzichini MM, Gibson P, et al. Sputum eosinophilia predicts benefit from prednisone in smokers with chronic obstructive bronchitis. Am J Respir Crit Care Med 1998; 158: 1511-1517.

10 Leigh R, Pizzichini MM, Morris MM, et al. Stable COPD: predicting benefit from high-dose inhaled corticosteroid treatment. Eur Respir J 2006; 27: 964-971.

11 Barnes NC, Sharma R, Lettis S, et al. Blood eosinophils as a marker of response to inhaled corticosteroids in COPD. Eur Respir J 2016; 47: 1374-1382.

12 Bafadhel M, McKenna S, Terry S, et al. Blood eosinophils to direct corticosteroid treatment of exacerbations of chronic obstructive pulmonary disease: a randomized placebo-controlled trial. Am J Respir Crit Care Med 2012; 186: 48-55.

13 Lee J, Machin M, Russell KE, et al. Corticosteroid modulation of immunoglobulin expression and B-cell function in COPD. FASEB J 2016; 30: 2014-2026.

14 Bourbeau J, Christodoulopoulos P, Maltais F, et al. Effect of salmeterol/fluticasone propionate on airway inflammation in COPD: a randomised controlled trial. Thorax 2007; 62: 938-943.

15 Ballarin A, Bazzan E, Zenteno RH, et al. Mast cell infiltration discriminates between histopathological phenotypes of chronic obstructive pulmonary disease. Am J Respir Crit Care Med 2012; 186: 233-239. 